\title{
AN INFORMATION THEORETIC METHOD OF MICROARRAY PROBE DESIGN FOR GENOME CLASSIFICATION
}

\author{
Elaine Garbarine and Gail Rosen \\ emg26@drexel.edu, gailraece.drexel.edu \\ Department of Electrical and Computer Engineering \\ Drexel University \\ Philadelphia, PA 19130
}

\begin{abstract}
In recent years, oligo microarrays, or more commonly-known DNA chips, have had a major impact in disease diagnosis, drug discovery, and gene identification. Microarrays contain Nmer DNA fragments, or oligos, in a series of "wells" placed across the chip, where each well contains thousands of the same fragments and acts as a probe that detects the amount of a specific fragment. A recent use for microarrays is for identification of genomes, such as pathogens. In current techniques, probes that detect unique gene regions of particular species are selected to be placed on the microarray, using the assumption that if one gene unique to a pathogen species can be detected, then the pathogen can be classified. This approach is useful, but the technology relies on finding the gene sequences that are divergent enough to be used as a genomic identifier and robust to cross-hybridization. In our work, we present a method to choose the most unique probes between two organisms. We accomplish this by choosing the oligo probes that maximize the level of divergence between the genomes, calculated by three different information-theoretic measures. We show the results for a 12-mer and 25mer oligo pathogen probe set and that our method chooses probes less likely to cross-hybridize.
\end{abstract}

\section{Index Terms - DNA, Information Theory, Microorganisms}

\section{MICROARRAYS AND PATHOGEN DETECTION}

The number of published sequenced genomes has been exponentially increasing since 1995, with over 600 completed by 2007 [1]. This makes an expanding database for genome analysis, including phylogeny (evolutionary tree) studies, comparative gene analysis, etc. A new application using apriori genome databases is to characterize genomic fingerprints to identify a particular genome, especially if it is a pathogen. In the field of metagenomics, the study of genetic material recovered from environmental samples, scientists now have the potential to classify a species based on genomic features and compare it to signatures found in a database rather than using taxonomy and classical phenotypic features.

The two major methods for identifying pathogens are PCR (polymerase chain reaction) assays and microarray chips. In PCR methods, slight variations unique to certain genomes, such as SNPs (single nucleotide polymorphisms), are examined to characterize particular pathogens. Unfortunately, in genomes with high mutations, such as HIV virii, PCR needs more than a few identifiers [2]. Many pathogens contain mobile genetic elements that can potentially interfere with proper identification using single loci detection systems and therefore, many loci are needed.With the advent of microarrays, there have been several methods developed [3, 4, 5]. In [4], each probe selected is a substring of a gene, which acts as its fingerprint. Their Findprobe program takes only genes as input and designs probes which satisfy homogeneity, sensitivity, and specificity constraints. In [5], a highly redundant probe pair is placed on the chip for each diagnostic region specified. Almost all previous microarray classification methods use ways to detect particular genomic regions rather than using the genome sequence statistics and built-in Nmer signatures. Only recently, in [3], have shorter Nmers (12/13-length) been proposed for microarray placement detection, but since the $\mathrm{N}$-mers were sequence-independent, this research did not go further.

With the volume of genomes being sequenced, we can now develop genomic fingerprints based on the composition and develop information theoretic measures can to discriminate and classify particular species. Information theoretic measures have recently been shown useful to construct evolutionary histories of genomes [6]. In this paper, the novelty of our approach is that we can develop infotheoretic measures that utilize the full genome sequence, and are not only restricted to genes (or protein-coding sequences). Our technique uses the "full vocabulary" of the genome to design unique identifier probes that are useful for genomes that have a large percentage of noncoding regions.

The examples in this paper illustrate the design of probes to distinguish between a pair of genomes for use with microarrays that hybridize $N$-length oligos, or Nmers. Microarrays contain oligos in a series of "wells" placed across the chip, where each well contains thousands of the identical oligos and acts as a probe that detects the amount of a specific fragment. In this paper, we examine short sequences - a typical off-the-shelf microarray (such as Affymetrix) uses 25-length sequences. These are called "short" sequences since most microarrays measure gene expression, and the average length of a gene in bacteria is about $1000 \mathrm{bp}$ [7]. We will show that by using information theory design to maximize the entropy between Nmer probes, our method functions well even with shorter probe lengths, such as 12 mers.

\section{THE PROBE SELECTION PROCESS}

The goal of our design is to select Nmer probes that will efficiently discriminate between a pair of pathogens and adhere to thermodynamic constraints of microarrays. The affinity of some probes to hybridize over others is effected by the $\mathrm{C}+\mathrm{G}$ content and \% identity of sequences.

In addition to these constraints, we develop information-theoretic measures that maximize the probability of choosing a probe which is abundant in genome A while non-abundant in genome B. We select probes that best distinguish between genomes by maximizing the divergence and mutual information between the probe and genome classes. This is accomplished by intersecting the best probes in each measure.

Fig. 1 illustrates the probe selection process with each of the 


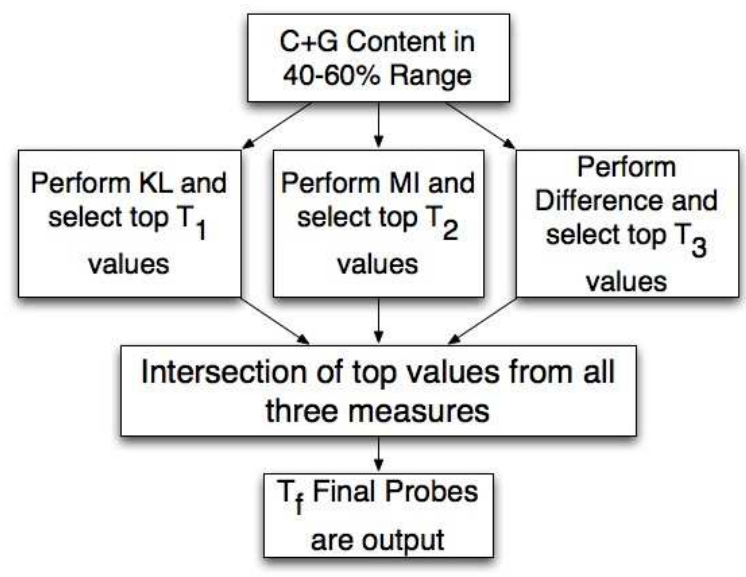

Fig. 1. Algorithm flow. Three measures used are Kullback-Leibler (KL), Mutual Information (MI) and Difference Measure (difference)

steps of the process detailed below. It is important to note that all measures are performed on a pair of genomes such that one pathogen can be identified from another pathogen.

\subsection{Genomic Signatures using Sequenced DNA}

Currently, microarray probes are designed to detect specific genes. This can become problematic because genes can occur at multiple points in the genome. So, microarrays may not have the specificity to detect a particular gene associated with an organism. We propose a design that examines the full genomic "vocabulary" of an organism by counting all the substrings, or Nmers, in the DNA sequence. This develops a genomic profile that can be used to characterize an organism. Figures 2 and 3 illustrate the profiles for $\mathrm{C}$. Michagenesis and $\mathrm{H}$. Influenzae for an Nmer length of $N=12$.

\subsection{Measures for Probe Selection}

The first important step in the probe selection process is the restriction on the $\mathrm{C}+\mathrm{G}$ content of the probe. The measure of $\mathrm{C}+\mathrm{G}$ content is the percentage of the sequence that is constructed of the cytosine and guanine bases. It has been shown that probes with high $\mathrm{C}+\mathrm{G}$ content need a higher temperature to hybridize which biases hybridization towards low $\mathrm{C}+\mathrm{G}$ content sequences. To assure an even hybridization we choose probes in the $40-60 \% \mathrm{C}+\mathrm{G}$ content range. The computation for the $\mathrm{C}+\mathrm{G}$ content of all of the probes must only be performed once and as such, it is done before the rest of the algorithm is performed.

Following the $\mathrm{C}+\mathrm{G}$ content check, three measures are computed to maximize divergence between probes for genome discrimination:

Difference in Nmer counts: This step simply calculates the difference between Nmer probe counts present in both sequences. If $X_{A}(m)$ represents the Nmer count for the $m$ th nmer for pathogen $\mathrm{A}$ and $X_{B}(m)$ represents the counts for the $m$ th Nmer for pathogen $\mathrm{B}$, we compute the difference metric as follows:

$$
D(n)=\left|X_{A}(m)-X_{B}(m)\right|
$$

This generates a measure of the difference in Nmer levels between two pathogens.

Kullback-Leibler Divergence: Given genome A and genome B, we can compute the Kullback-Leibler divergence for the $m$ th Nmer between each genome in the set $\mathbf{C}=\{\mathbf{A}, \mathbf{B}\}$. The probability of the $m$ th Nmer in genome set $\mathbf{C}$ represented by $p_{C}(m)$ :

$$
\begin{aligned}
& \mathbf{D}_{\mathbf{K L}}(A(m), B(m))= \\
& \quad p_{A}(m) \log _{2} \frac{p_{A}(m)}{p_{B}(m)}+p_{B}(m) \log _{2} \frac{p_{B}(m)}{p_{A}(m)}
\end{aligned}
$$

where $\mathbf{D}_{\mathbf{K L}}$ is the symmetric Kullback-Leibler distance and $p_{C}(m)$ is calculated by the amount of the $m$ th Nmer in genome set $\mathbf{C}, X_{c}(m)$, divided by the total number of Nmers in the genome.

Mutual Information(MI): To derive the mutual information for an Nmer and associated genomes, we have the genomes, $\mathbf{C}$, and an $M$-dimensional feature space

$\mathbf{X}_{\mathbf{C}}=\left\{X_{C}(1), X_{C}(2), X_{C}(3), \ldots X_{C}(M)\right\}$ where each $X_{C}(m)$ represents the amount of the $m$ th Nmer present in a genome C. In our problem, we want to find the Nmers with the maximum MI between the Nmer and the set of genomes, $I(X(m), \mathbf{C})$ which are the $X(m)$ 's that satisfy:

$$
\arg \max _{X(m)} I(X(m), \mathbf{C})=\arg \max \sum_{c \in \mathbf{C}} p\left(X_{C}(m), c\right) \log _{2} \frac{p\left(X_{C}(m), c\right)}{p\left(X_{C}(m)\right) p(c)}
$$

MI can be rewritten as $I(X(m), \mathbf{C})=H(\mathbf{C})-H(\mathbf{C} \mid X(m))$. Since the conditional entropy of the genomes given an Nmer, $H(\mathbf{C} \mid X(m))$, is always negative, $I(X(m), \mathbf{C})$ is maximized by maximizing $H(\mathbf{C} \mid X(m))$ since the marginal entropy of the genomes, $H(\mathbf{C})$, is constant. Therefore, the best Nmers that discriminate between genomes can be interpreted as the ones with the highest conditional entropy.

The Nmers that maximize each measure are sorted from highest to lowest. Then, the Nmers that maximize all these requirements are chosen. This method eliminates Nmer probes that have similar counts in each genome which is crucial since these probes have low information content.

Probes should also be checked to minimize cross-hybridization. Under appropriate experimental conditions (i.e., temperature and salt concentration), perfect match fragments will hybridize to the microarray probes (bind to the complementary capturing oligos). Nevertheless, there will always be some nonspecific bindings since fragments may cross-hybridize to probes that are not the perfect match but have some mismatches. Studies have shown that microarry crosshybridization becomes a problem if sequences contain $70 \%$ similar bases [8] [9]. One way to verify if sequences are less than $70 \%$ identical is to measure the hamming distance between the probes.

A Hamming distance between two strings of equal length is described as the number of positions for which corresponding symbols are different. We denote the Hamming Distance between two probes, $x$ and $y$ as $H_{x y}$ and define it as: $H_{x y}=\sum_{i=1}^{N}\left(x_{i} \neq y_{i}\right)$ where $i$ denotes the $i$ th position in the probe and $N$ denotes the total length of the probe. In the next section, we show that the method naturally selects hamming-distant probe sets, and thus, the information-theoretic measures meet this requirement.

\section{EXAMPLE OF PROBE SELECTION BETWEEN TWO PATHOGENS}

\subsection{Data Acquisition}

The data in this paper is obtained from The Sanger Institute [10]. The Sanger Institute has ongoing sequencing projects aimed at fully 
sequencing human and genome pathogens. Currently, a 32 out of a total 92 bacterial pathogen sequencing projects are completed. We select an a pair of genomes to test our algorithm: Clavibacter Michiganensis and Haemophilus Influenzae.

\subsection{Results}

As described in Section 2.2 the $\mathrm{C}+\mathrm{G}$ content of each word is checked and all words not within the range of $40-60 \%$ are discarded. Following this step, the desired number of output probes, $T_{f}$, is chosen. We select $80 \leq T_{f} \leq 100$ to yield an example set. A search is performed on the KL/MI/difference thresholds, $T_{1}, T_{2}$, and $T_{3}$, to obtain the desired $T_{f}$ output probes. These thresholds can be adjusted to vary the weight of each measure on the outputs and to select the $T_{f}$ outputs desired.

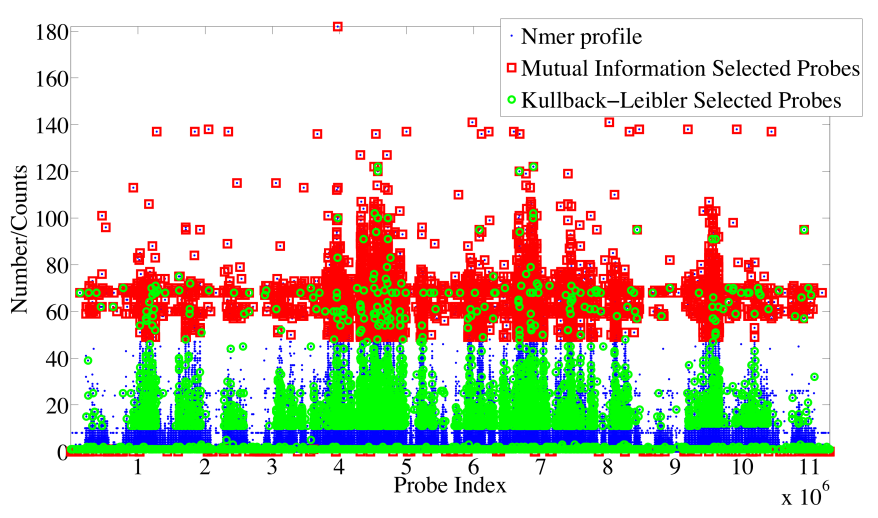

Fig. 2. The $12 \mathrm{mer}$ profiles and probe selection for C. Michagenesis. The blue-starred line signifies all Nmer counts in each of the organisms. The red squares represent the probes selected based on the MI measure with $T_{1}=$ 5000 , and the green circles represent the probes selected based on the KL measure with $T_{2}=5000$. The mutual information measure chooses probes that are numerous or near non-existent. The final probes are the overlap of the KL/MI/DV measures.

In our case of $N=12$ length words, the KL and MI thresholds ( $T_{1}$ and $T_{2}$ ) are chosen to be 5000 . If $T_{1}, T_{2}$ values under 5000 are used, only 20 finals probes are output due to few overlapping probes between the MI and KL measures as can be seen in Figs. 2 and 3. Due to $T_{1}$ and $T_{2}$ being the limiting factors, a higher value of $T_{3}=10000$ is set to ensure enough distance between the Nmer counts.

Taking the intersection of these three measures for $N=12$ results in 86 common probes. Fig. 4 displays the levels of each specific probe in each of the two genomes.

The same method is performed again for the two pathogens but now for $N=25$, or 25mers. $T_{1}$ and $T_{2}$ are set to 85000 to yield enough overlapping MI and KL probes and $T_{3}$ is set to 19650 to ensure enough Nmer level distance between the two. The thresholds are notably higher in the $N=25$ over the $N=12$ case. This is due to the fact that there are less 25 mers on average since the genome length stays constant, but now the number of substrings counted are spread over $4^{25}$ combinations as opposed to $4^{12}$. For $N=25$, the intersections of the three measures yield a final 101 probes. Fig. 5 displays the amount of each probe for the two genomes.

For each of the two Nmer lengths described above, the final probes are subjected to a check of Hamming distance to detect potential cross-hybridization. The Hamming distance is computed for all possible combinations of the final selected probes. As discussed in

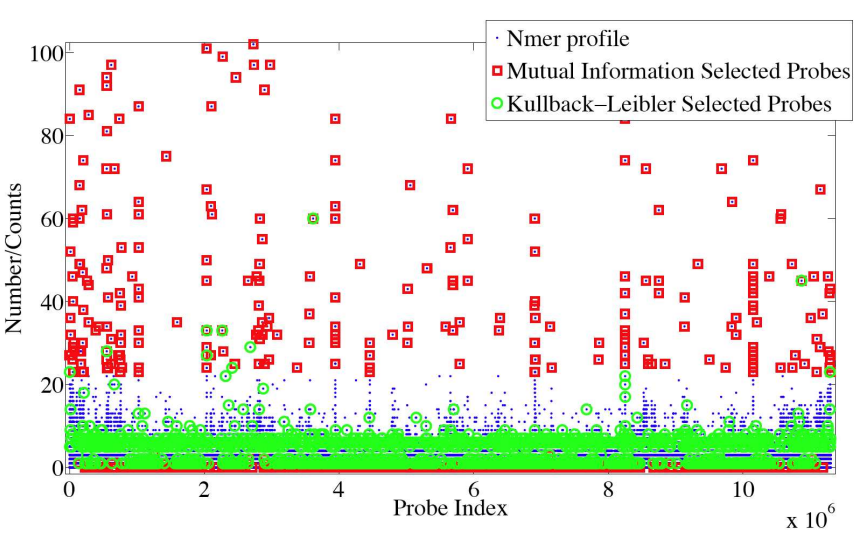

Fig. 3. The 12 mer profiles and probe selection for $H$. Influenzae. The blue-starred line signifies all Nmer counts in each of the organisms. The red squares represent the probes selected based on the MI measure with $T_{1}=5000$, and the green circles represent the probes selected based on the KL measure with $T_{2}=5000$. The mutual information measure tends to choose probes that are numerous or near non-existent while K-L chooses probes with a consistent separation. Intersecting the measures yields probes that are highly divergent with consistent separation, and very few probes meet this strict criteria.

section 2, two sequences with greater than $70 \%$ similar bases would be extremely likely to cross hybridize thus we want hamming distances that are high to minimize this effect. In Figs. 6 (a) and 6 (b), it is shown that almost all combinations of probes have hamming distances that reflect less than 70\% similar bases for both 12 and 25 length words. This demonstrates that our method in fact selects probes that are sufficiently non-specific in binding to avoid the problem of cross hybridization that plagues microarrays.

The algorithm can be run on any desktop computer. Our example can run in a little over an hour on an Apple G5: 20 minutes for the Nmer counting in each pathogen, 10 minutes for the $\mathrm{C}+\mathrm{G}$ content, 30 minutes for the Information measures, and 10 minutes for the Hamming distance crosscheck.

\subsection{Extending to larger mixtures}

While we illustrate a method to distinguish between a pair of genomes, in practice, pathogen detection applications need to detect an agent out of thousands of potential pathogens. One way to accomplish this is to compare all combinations of pathogens in pairwise fashion in order to develop a common probeset. To illustrate this idea we take the probes discussed earlier from the pairwise comparison of $\mathrm{C}$. Michiganensis and $\mathrm{H}$. Influenzae with word length of $N=12$ which produced 86 . In addition we perform the algorithm on a different set of pathogens, namely C. Michiganensis and E. Shigella also using the word length $N=12$, producing 109 final probes. The intersection of these two sets of probes is then performed and we find a final results of 37 probes common in each set. A classifier could then be formed from these 37 probes with varying thresholds set for each Nmer such that it can discriminate one pathogen from a mixture of the three. This concept could be extended further to find common probesets and produce binary classifiers for each Nmer feature thus allowing us to work with a much larger genome set. With further development, we plan to test the probe performance on Affymetrix and shorter-oligo microarrays. 


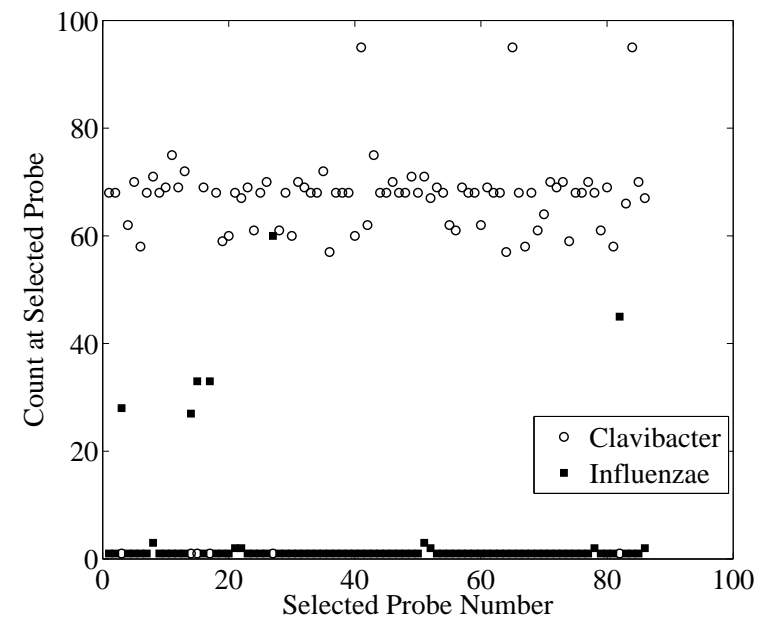

Fig. 4. Comparison of the final selected probes counts for the pathogen pair of C. Michiganensis and H. Influenzae with word length $N=12$. The thresholds for each step in Fig. 1 were: $T_{1}=5000, T_{2}=5000$, $T_{3}=100000$. This computation yielded 86 final probes. A linear best fit to each curve shows that the method chooses the probes that are abundant in $\mathrm{C}$. Michiganensis but almost nonexistent in H. Influenzae.

\section{CONCLUSIONS}

In this paper, we develop an information-theoretic method that exploits genome vocabularies to distinguish between pathogens. Three measures (MI, K-L divergence, and Difference Values) are computed on Nmer word counts of pathogens, and the intersection of the probes that maximize each measure are used to identify the top $T_{f}$ final probes. We illustrate that the resulting 12-mer and 25-mer oligo probe sets effectively distinguish between pathogens due to Nmer copy number variation. Also, we show that shorter oligo probesets yield less number of probes but greater distinction than longer oligo probesets. Finally, the oligo probes chosen are shown to have sufficiently large Hamming distances to prevent cross-hybridization.

\section{ACKNOWLEDGEMENTS}

We would like to thank Christopher Pearson of Pearson Consulting in Minneapolis, $\mathrm{MN}$ for his valuable work in computing the Nmer counts and signature profiles of the pathogens.

\section{REFERENCES}

[1] http://www.genomesonline.org/, "Gold (genomes online database)," .

[2] Gardner et al., "Limitations of taqman per for detecting divergent viral pathogens illustrated by hepatitis a, b, c, and e viruses and human immunodeficiency virus," Journal of Clinical Microbiology, 2003.

[3] Y. Y. et al. Belosludtsev, "Organism identification using a genome sequence-independent universal microarray probe set," BioTechniques, 2004.

[4] W-K Sung, "Fast and accurate probe selection algorithm for large genomes," in IEEE Proceedings of the Computational Systems Bioinformatics, 2003.

[5] W. J. et al. Wilson, "Sequence-specific identification of 18 pathogenic microorganisms using microarray technology," Molecular and Cellular Probes, vol. 16, pp. 119-127, 2002.

[6] P. et al. Hanus, "Information theoretic distance measuers in phylogenomics," in International Workshop on Information Theory and Applications (ITA), 2007.

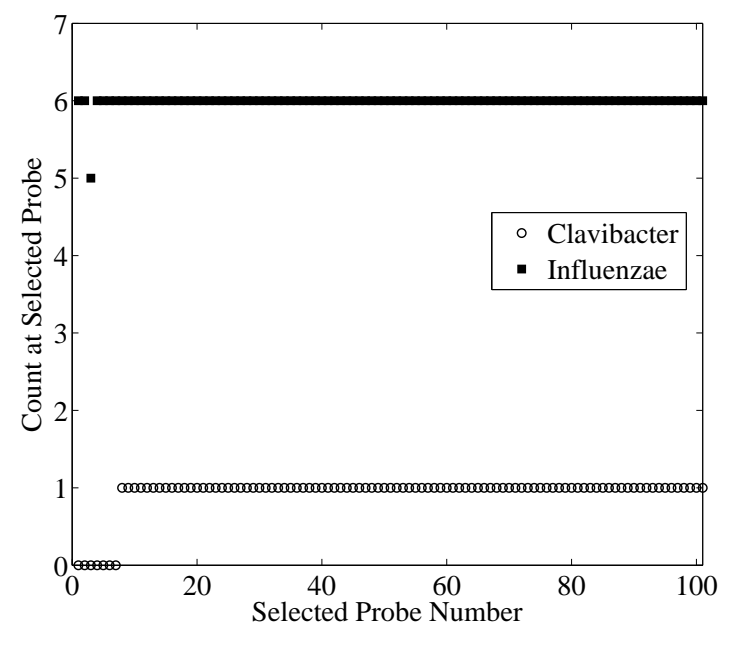

Fig. 5. Comparison of the final selected probes counts for the pathogen pair of C. Michiganensis and $\mathrm{H}$. Influenzae with word length $N=25$. The thresholds for each step in Fig. 1 were: $T_{1}=85000, T_{2}=85000, T_{3}=$ 19650. This computation yielded 101 final probes. A linear best fit to each curve shows the divergence in the chosen genome counts.

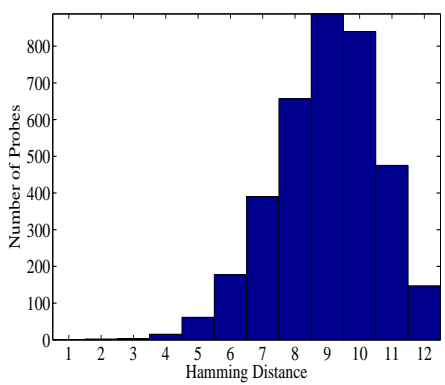

(a)

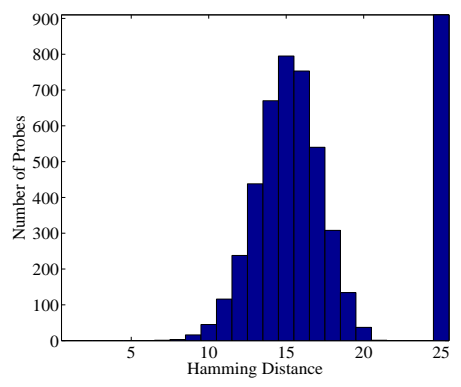

(b)
Fig. 6. (a) Histogram of Hamming distances for all combinations of the final 86 probes for the pathogen pair C. Michiganensis and $\mathrm{H}$. Influenzae with word length, $N=12$. These Hamming Distances have mean $=8.9759$ and standard deviation $=1.6063$, thus probes are only $26 \%$ identical on average and only 5 out of a possible 3655 combinations of probes are greater than $70 \%$ identical. (b) Hamming distance for all combinations of the final 101 probes for the same pathogen pair with word length, $N=25$. These Hamming Distances have mean $=16.9001$ and standard deviation $=4.2422$, thus probes are only only $32.4 \%$ identical on average and 1 out of a possible 5050 combinations of probes are greater than $70 \%$ identical.

[7] J. Zhang, "Protein-length distributions for the three domains of life," Trends in Genetics, vol. 16, pp. 107-109, 2000.

[8] Michael D. Kane, Timothy A. Jatkoe, Craig R. Stumpf, Jia Lu, Jeffrey D. Thomas, and Steven J. Madore, "Assessment of the sensitivity and specificity of oligonucleotide (50mer) microarrays," Nucl. Acids Res., vol. 28, no. 22, pp. 4552-4557, 2000.

[9] T.R. Hughes and et al., "Expression profiling using microarrays fabricated by an ink-jet oligonucleotide synthesizer.," Nat Biotechnol, vol. 19, no. 4, pp. 342-7, 2001.

[10] http://www.sanger.ac.uk/Projects/Microbes/, "Sanger institute sequencing project," 